\title{
Fauna of Protected Areas - 24: SCARABAEID BEETLES OF BANDHAVGARH NATIONAL PARK, MADHYA PRADESH
}

\author{
Kailash Chandra and S.C. Ahirwar
}

Central Regional Station, Zoological Survey of India, Jabalpur, Madhya Pradesh 482002, India

Email: crszsijb@sancharnet.in

\begin{abstract}
A comprehensive survey of Bandhavgarh National Park in Madhya Pradesh revealed 44 species in 24 genera and eight subfamilies. Thirty-five species are first time records for Bandhavgarh National Park and Umaria district, and five species are new records for Madhya Pradesh state.
\end{abstract}

\section{Keywords}

Bandhavgarh National Park, checklist, new records, scarabeid beetles

Bandhavgarh National Park is an important and old national park in Madhya Pradesh. An area of $105 \mathrm{~km}^{2}$ was declared as National park in 1965 and it was finally notified in 1968 (vide Forest Department notification No. 2977/X/68 dated 23.iii.1968). The park is located about $130 \mathrm{~km}$ northeast of Jabalpur and $30 \mathrm{~km}$ from Umaria. The park lies between $80^{\circ} 57^{\prime} 30^{\prime \prime}-80^{\circ} 06^{\prime} 15^{\prime \prime} \mathrm{E}$ $\& 23^{0} 36^{\prime} 30^{\prime \prime}-23^{\circ} 42^{\prime} 30^{\prime \prime} \mathrm{N}$. The altitude ranges from $440 \mathrm{~m}$ in the valleys to $811.40 \mathrm{~m}$ on the highest ridge top. The area consists of many hills and hillocks of varying sizes interspersed with large number of various-sized valleys. Most of the area is undulating, however, the hill tops are flat. The lower portions of the valleys are grassy meadows locally called bah, which are mostly marsh land with seepage from ground. The vegetation of the park is tropical moist deciduous type, which may be reclassified into Sal forest, mixed forests and grasslands.

The family Scarabaeidae is one of the largest families in the order Coleoptera, which have both positive as well as negative significance. Dung beetles play an important role in enhancing the nutritional value of the soil, whereas chafers feed on vegetation. The first comprehensive account of Scarabaeid beetles of the Indian region was published by Arrow (1910, 1917, 1931) in three volumes of Fauna of British India and 58 species were reported from Madhya Pradesh, wherein only nine species of the subfamily Scarabaeinae were known from Umaria district. Some other papers on Scarabaeid beetles of Madhya Pradesh and Chhattisgarh were published by Chandra (2000), and, Chandra and Singh (2004), but the information on scarabaeid beetles of Bandhavgarh National Park is completely lacking. During the survey of Bandhavgarh National Park undertaken by the Zoological Survey of India in 2002 and 2004, several specimens of scarabaeid beetles were studied, which resulted in the identification of 44 species of 24 genera under eight subfamilies. Thirty-five species are recorded for the first time from Bandhavgarh National Park and Umaria district. Of these, five species are new records to the fauna of Madhya Pradesh. All the species are identified by the senior author and the identified specimens are registered and deposited in the National Zoological Collection of Zoological Survey of India, Jabalpur.
List of scarabaeid beetles from Bandhavgarh National Park

\author{
Family: Scarabaeidae \\ Subfamily: Aphodinae \\ 1. Aphodius moestus Fabricius \\ 2. Aphodius crenatus Harold
}

Subfamily: Hybosridae

3. Hybosorus orientalis Westwood

4. Phaeochrous sp.

\section{Subfamily: Troginae}

5. Trox sp.

\section{Subfamily: Scarabaeinae}

6. Catharsius pithecius (Fabricius)

7. Catharsius molossus (Linnaeus)

8. Catharsius sagax (Queensland)

9. Copris imitans Felsche *

10. Copris repertus Walker*

11. Drepanocerus setosus (Wiedemann)*

12. Gymnopleurus sinuatus (Olivier)

13. Heliocopris bucephalus (Fabricius)

14. Liatongus rhadamistus (Fabricius)*

15. Onthophagus bonasus (Fabricius)

16. Onthophagus catta (Fabricius)

17. Onthophagus cervus (Fabricius)*

18. Onthophagus dama (Fabricius)

19. Onthophagus fasciatus Boucomont *

20. Onthophagus ramosus (Wiedman)

21. Onthophagus pactolus (Fabricius)

22. Onthophagus quadridentatus (Fabricius)*

23. Onthophagus sagittarius (Fabricius)

24. Onthophagus tarandus (Fabricius) *

25. Onitiicellus cintus (Fabricius)

26. Oniticellus spinipes Roth*

27. Onitis philemon (Fabricius)

\section{Subfamily: Melolonthinae}

28. Apogonia sp.

29. Schizonycha ruficollis (Fabricius)

\section{Subfamily: Rutelinae}

30. Adoretus bicaudatus Arrow

31. Adoretus lasiopygus Burmeister

32. Adoretus duvauceli Blanchard

33. Mimela inscripta (Nonfried)

34. Mimela macleayana (Vigors)

35. Rhinyptia meridionalis Arrow

36. Singhala robusta (Ohaus)

37. Pachyrrhinadoretus frontatus (Burm.)

\section{Subfamily: Dynastinae}

38. Heteronychus lioderes Redtenbacher

39. Alissonotum simile Arrow

40. Dipelicus cantator Arrow

41. Xylotrupes gideon (Linnaeus) 


\section{Subfamily: Cetoniinae}

42. Clinteria klugi (Hope)

43. Chiloloba acuta (Wied.)

44. Oxycetonia versicolar (Fabricius)

\section{Systematic Account}

Order: Coleoptera

Suborder: Polyphaga

Family: Scarabaeidae

Subfamily: Aphodiinae

1. Aphodius moestus Fabricius

1801. Aphodius moestus Fabricius, Syst. Eleuth., 1: 78.

1964. Aphodius moestus Balthasar, Verl. Tschsl. Akad. Wissensch. III: 182.

Material examined: Umaria, B.N.P., Tala Guest house, 28.ix.2002 (2 exs.), coll. R.K. Singh \& party.

Remarks: Very common; new record from Bandhavgarh National Park.

\section{Aphodius crenatus Harold}

1862. Aphodius crenatus Harold, Berl. Ent. Zeit.,5, 1862, p.14, 142. 1898. Aphodius crenatus Cloust, Ann. Soc. Ent. Fr., 67, p.240. Material examined: Umaria, B.N.P., Tala Guest house, 22.ix.2002 (1 ex.), coll. M.L. Koshta \& party; 30.ix.2002 (1ex.), coll. R.K. Singh \& party.

Remarks: Very common; new record from Bandhavgarh National Park

\section{Subfamily: Hybosorinae}

3. Hybosorus orientalis Westwood

1845. Hybosorus orientalis Westwood, Trans. Ent. Soc. Lond., 6: 159.

1983. Hybosorus orientalis Kuitjen, Zool. Varh. Leiden, 203: 25. Material examined: Umaria, B.N.P., Tala Guest house, 21.ix.2002 (1 ex.), 22.ix.2002 (2 exs.), coll. M.L. Koshta \& party.

Remarks: Common; new record from Bandhavgarh National Park.

\section{Phaeochrous sp.}

1840. Phaeochrous Castelnau, Hist. nat. des. Insectes. 2(1): 108.

1983. Phaeochrous Kuitjen, Zool. Verh. Leiden, 165: 8.

Material examined: Umaria, B.N.P., Tala Guest house, 23.ix.2002 (1 ex.), coll. M.L. Koshta \& party.

Remarks: Common; new record from Bandhavgarh National Park.

\section{Subfamily: Troginae}

5. Trox sp.

1792. Trox Fabricius, Syst. Ent., 31.

1912. Trox Arrow, Col. Cat. Pars, 43: 53.

1982. Trox Scholtz, Ent. Mem. 54: 2.

Material examined: Umaria, B.N.P., Tala Guest house, 22.ix.2002 (1 ex.), coll. M.L. Koshta \& party.

Remarks: Common; new record from Bandhavgarh National Park.

\section{Subfamily: Scarabaeinae}

6. Catharsius pithecius (Fabricius)

1775. Scarabaeus pithecius Fabricius, Syst. Ent.,: 21.

1931. Catharsius pithecius Arrow, Faun. Brit. India (Lamellicornia: Coprinae) 3: 100

Material examined: Umaria, B.N.P., Tala Guest House, 24.ix.2002 (2 exs.), 25.ix.2002 (2 exs.), 28.ix.2002 (2 exs.) \& 29.ix.2002 (1 ex.), coll. M.L. Koshta \& party.

Remarks: Very common; new record from Bandhavgarh National Park.

7. Catharsius molossus (Linnaeus)

1758. Scarabaeus molossus Linnaeus, Syst. Nat. ed. 10:347.

1931. Catharsis molossus Arrow, Faun. Brit. India, (Lamellicornia: Coprinae) 3: 95-96.

Material examined: Umaria, B.N.P., Tala Guest house, 25.ix.2002 (10 exs.), 27.ix.2002 (2 exs.) \& 28.ix.2002 (10 exs.), coll. M.L. Koshta \& party; Bamera dam, 1.x.2002 (3 exs.); Tala Guest house, 3.x.2002 (8 exs.), coll. R.K. Singh \& party.
Remarks: Very common; new record from Bandhavgarh National Park.

8. Catharsius sagax (Queensland)

1806. Copris sagax Queensland, Schonh. Syn. Ins., I: 43.

1931. Catharsius sagax: Arrow, Faun. Brit. India (Lamellicornia: Coprinae) 3: 96.

Material examined: Umaria, B.N.P., Tala Guest house, 25.ix.2002 (4 exs.), 27.ix.2002 (2 exs.) \& 28.ix.2002 (1ex.), coll. M.L. Koshta \& party.

Remarks: Very common; new record from Bandhavgarh National Park.

\section{Copris imitans Felsche}

1910. Copris imitans Felsche, Deutsche Ent. Zeits., 347.

1931. Copris imitans: Arrow, Faun. Brit. India, (Lamellicornia: Coprinae) 3: 124.

Material examined: Recorded from Literature (Arrow, 1931)

Remarks: Common; known from Umaria district.

10. Copris repertus Walker

1858. Copris repertus Walker, Ann. Mag. Nat. Hist., (3)ii: 208.

1931. Copris repertus Arrow, Faun. Brit. India, (Lamellicornia: Coprinae) 3: 116 .

Material examined: Recorded from literature (Arrow, 1931)

Remarks: Common; known form Umaria District.

11. Drepanocerus setosus (Wiedemann)

1823. Copris setosus Wiedemann, Zool. Mag., II, i: 19.

1931. Drepanocerus setosus Arrow, Faun. Brit. India, (Lamellicornia:

Coprinae) 3: 381 .

Material examined: Recorded from literature (Arrow, 1931)

Remarks: Common; known from Umaria district.

\section{Gymnopleurus sinuatus (Olivier)}

1789. Scarabaeus sinuatus (Olivier), Entom., I: 160, tf..21, fig.189. 1931. Gymnopleurus sinuatus Arrow, Faun. Brit. India, (Lamellicornia: Coprinae) 3:63.

Material examined: Umaria, B.N.P., Tala Guest house, 22.ix.2002 (1 ex.), coll. M.L. Koshta \& party.

Remarks: Common; new record from Bandhavgarh National Park.

13. Heliocopris bucephalus (Fabricius)

1775. Scarabaeus bucephalus Fabricius, Syst. Ent., 24

1931. Heliocopris bucephalus Arrow, Faun. Brit. India, (Lamellicornia: Coprinae) 3:88.

Material examined: Umaria, B.N.P., Tala Guest house, 27.ix.2002 (3 exs.), 28.ix.2002 (2 exs.), coll. M.L. Koshta \& party.

Remarks: Very common; new record from Bandhavgarh National Park.

14. Liatongus rhadamistus (Fabricius)

1775. Scarabaeus rhadamistus Fabricius, Syst. Ent., 22.

1931. Liatongus rhadamistus: Arrow, Faun. Brit. India, (Lamellicornia: Coprinae) 3:374

Material examined: Recorded from literature (Arrow, 1931)

Remarks: Common; known from Umaria district.

15. Onthophagus bonasus (Fabricius)

1775. Scarabaeus bonasus Fabricius, Syst. Ent., : 23.

1931. Onthophagus bonasus: Arrow, Faun. Brit. India, (Lamellicornia: Coprinae) 3:231.

Material examined: Umaria, B.N.P., Tala Guest house, 22.ix.2002 (1 ex.), coll. M.L. Koshta \& party.

Remarks: Common; new record from Bandhavgarh National Park.

16. Onthophagus catta (Fabricius)

1787. Scarabaeus catta Fabricius, Mant. Ins., i: 12.

1931. Onthophagus catta: Arrow, Faun. Brit. India, (Lamellicornia: Coprinae) 3:230.

Material examined: Umaria, B.N.P., Tala Guest house, 22.ix.2002 (1 ex.), 24.ix.2002 (2 exs.) \& 26.ix.2002 (1 ex.), coll. M.L. Koshta \& 
party.

Remarks: Common; new record from Bandhavgarh National Park.

17. Onthophagus cervus (Fabricius)

1798. Copris cervus F., Ent. Syst. Suppl., : 31.

1931. Onthophagus cervus Arrow, Faun. Brit. India, (Lamellicornia: Coprinae) 3:348.

Material examined: Umaria, Tala Guest house, 24.ix.2002 (2 exs.), coll. M.L. Koshta \& party.

Remarks: Common; known from Umaria district.

18. Onthophagus dama (Fabricius)

1798. Copris dama Fabricius, Ent. Syst. Suppl., 32.

1931. Onthophagus dama: Arrow, Faun. Brit. India, (Lamellicornia: Coprinae) 3:280.

Material examined: Umaria, B.N.P., Tala Guest house, 24.ix.2002 (2 exs.), coll. M.L. Koshta \& party.

Remarks: Very common; new record from Bandhavgarh National Park.

19. Onthophagus fasciatus Boucomont

1914. Onthophagus fasciatus Boucomont, Ann. Mus. Civ. Genova, xlvi: 214.

1931. Onthophagus fasciatus: Arrow, Faun. Brit. India, (Lamellicornia: Coprinae) 3: 311

Material examined: Recorded from literature (Arrow, 1931).

Remarks: Common; known from Umaria district.

20. Onthophagus ramosus Wiedman

1823. Onthophagus ramosus (Wiedman), Zool. Mag., 21.

1931. Onthophagus ramosus: Arrow, Faun. Brit. India, (Lamellicornia: Coprinae) 3:236.

Material examined: Umaria, B.N.P., Tala Guest House, 29.ix.2002 (1 ex.), 30.ix.2002 (1 ex.), coll. R.K. Singh \& party.

Remarks: Very common; new record from Bandhavgarh National Park.

21. Onthophagus pactolus (Fabricius)

1787. Scarabaeus pactolus Fabricius, Mat. Ins., I: 12.

1931. Onthophagus pactolus: Arrow, Faun. Brit. India, (Lamellicornia: Coprinae) 3:203.

Material examined: Umaria, B.N.P., Tala Guest House, 24.ix.2002 (1 ex.), coll M.L. Koshta \& party.

Remarks: Common; new record from Bandhavgarh National Park.

22. Onthophagus quadridentatus (Fabricius)

1798. Copris quadridentatus Fabricius, Ent. Syst. Suppl., 34.

1931. Onthophagus quadridentatus Arrow, Faun. Brit. India, (Lamellicornia: Coprinae) 3: 282.

Material examined: Recorded from literature (Arrow, 1931)

Remarks: Common; known from Umaria district.

23. Onthophagus sagittarius (Fabricius)

1775. Copris sagittarius Fabricius, Syst. Ent., p. 21.

1931. Onthophagus sagittarius Arrow, Faun. Brit. India, (Lamellicornia: Coprinae) 3: 304.

Material examined: Umaria, B.N.P., Gomphari dam, 02.11 .2004 (2 exs.), coll. Y.N. Gupta \& party.

Remarks: Common; new record from Bandhavgarh National Park.

24. Onthophagus tarandus (Fabricius)

1792. Scarabaeus tarandus F., Ent. Syst., I: 48.

1931. Onthophagus tarandus: Arrow, Faun. Brit. India, (Lamellicornia:

Coprinae) 3:180.

Material examined: Umaria, B.N.P., Tala Guest house, 24.ix.2002 (2 exs.), coll. M.L. Koshta \& party; Tala Guest house, $30 . i x .2002$ (1 ex.), coll. R.K. Singh \& party.

Remarks: Common; known from Bandhavgarh National Park.

25. Oniticellus cinctus (Fabricius)

1775. Scarabaeus cinctus F., Syst. Ent., 30.
1931. Oniticellus cinctus: Arrow, Faun. Brit. India, (Lamellicornia: Coprinae) 3:379.

Material examined: Umaria, B.N.P., Tala Guest house, 24.ix.2002 (2 exs.), coll. M.L. Koshta \& party.

Remarks: Very common; new record from Bandhavgarh National Park.

\section{Oniticellus spinipes Roth}

1851. Oniticellus spinipes Roth., Arch. F. Nat., xvii, i:128.

1931. Oniticellus spinipes: Arrow, Faun. Brit. India, (Lamellicornia: Coprinae) 3:378.

Material examined: Recorded from Literature (Arrow, 1931)

Remarks: Very common; known from Bandhavgarh National Park.

\section{Onitis philemon Fabricius}

1801. Onitis philemon Fabricius, Syst. Eleut., I: 30.

1931. Onitis philemon: Arrow, Faun. Brit. India, (Lamellicornia: Coprinae) 3: 393.

Material examined: Umaria, B.N.P., Tala Guest house, 21.ix.2002 (1 ex.), 24.ix.2002 (1 ex.), coll. M.L. Koshta \& party.

Remarks: Common; new record from Bandhavgarh National Park.

\section{Subfamily: Melolobthinae}

28. Apogonia sp.

1818. Apogonia Kirby, Trans. Linn. Soc., XII: 401.

1904. Apogonia Pering, Trans. Roy. Soc., 5, Aust. Phil. Soc., XIII: 289.

Material examined: Umaria, B.N.P., Tala Guest house, 22.ix.2002 (2 exs.), 24.ix.2002 (2 exs.), coll. M.L. Koshta \& party.

Remarks: Common; new record from Bandhavgarh National Park.

\section{Schizonycha ruficollis (Fabricius)}

1781. Melolontha ruficollis Fabricius, Spec. Ins., 7:39.

1923. Schizonycha ruficollis: Arrow, Rec. Indian Mus., 25(2): 250.

Material examined: Umaria, B.N.P., Tala Guest house, 22.ix.2002 (2 exs.), coll. M.L. Koshta \& party.

Remarks: Common; new record from Bandhavgarh National Park.

\section{Subfamily: Rutelinae}

\section{Adoretus bicaudatus Arrow}

1914. Adoretus bicaudatus Arrow, Ann, Mag. Nat. Hist., (8), xiii: 587. 1917. Adoretus bicaudatus Arrow, Faun. Brit. India, (Lamellicornia: Rutelinae) 2: 310.

Material examined: Umaria district, B.N.P., Tala Guest House, 29.ix.2002 (3 exs.), coll. R.K. Singh \& party.

Remarks: Very common; new record from Bandhavgarh National Park.

31. Adoretus lasiopygus Burmeister

1855. Adoretus lasiopygus Burmeister, Handb. Ent., 12(6): 530.

1910. Adoretus lasiopygus Arrow, Faun. Brit. India, (Lamellicornia: Rutelinae) 2: 349.

Material examined: Umaria, B.N.P., Tala Guest house, 24.ix.2002 (1 ex.), coll. M.L. Koshta \& party.

Remarks: Very common; new record from Bandhavgarh National Park.

\section{Adoretus duvauceli Blanchard}

1851. Adoretus duvauceli Blanchard, Cat. Coll. Ent. Mus. Paris, 432.

1917. Adoretus duvauceli Arrow, Faun. Brit. India, (Lamellicornia: Rutelinae) 2: 343.

Material examined: Umaria, Tala Guest house, 25.ix.2002 (1 ex.), 26.09.2002 (1 ex.), coll. M.L. Koshta \& party; Tala Guest house, 30.ix.202 (1 ex.), coll. R.K.Singh \& party.

Remarks: Very common; new record from Bandhavgarh National Park.

33. Mimela inscripta (Nonfried)

1892. Anomala inscripta Nonfried, Berl. Ent. Zeits., xxxvi, p. 236. 1917. Mimela inscripta: Arrow, Faun. Brit. India, (Lamellicornia: Rutelinae) 2: 124.

Material examined: Umaria, B.N.P., Tala Guest house, 24.ix.2002 (1 
ex.), coll. M.L. Koshta \& party.

Remarks: Rare; new record from Bandhavgarh National Park.

34. Mimela macleayana (Vigors)

1825. Euchlora macleayana Vigors, Zool. Journ., I: 540.

1910. Mimela macleayana Arrow, Faun. Brit. India, (Lamellicornia: Rutelinae) 2: 105

Material examined: Umaria, B.N.P., Tala Guest House, 24.ix.2002 (1 ex.), coll. M.L. Koshta \& party.

Remarks: Common; new record from Bandhavgarh National Park.

\section{Rhinyptia meridionalis Arrow}

1911. Rhinyptia meridionalis Arrow, Ann. Mag. Nat. Hist, (8) viii, p. 356.

1917. Rhinyptia meridionalis Arrow, Faun. Brit. India, (Lamellicornia: Rutelinae) 2: 271.

Material examined: Umaria, B.N.P., Tala Guest House, 22.ix.2002 (1 ex.), coll. M.L. Koshta \& party.

Remarks: Rare; new record from Madhya Pradesh

\section{Singhala robusta (Ohaus)}

1917. Anomala robusta Arrow, Faun. Brit. India, (Lamellicornia: Rutelinae) 2: 135.

1972. Singhala robusta Machatschke, Coleopteran Catalogue (W.Junk), Scarabaeoidea, Melolonthinae: Rutelinae: 194.

Material examined: Umaria, B.N.P, Tala Guest House, 22.ix.2002 (5 exs.), 24.ix.2002 (1 ex.), coll. M.L. Koshta \& party; Tala Guest house, 29.ix.2002 (1 ex.), 30.ix.2002 (2 exs.) \& 3.x.2002 (2 exs.), coll. R.K. Singh \& party.

Remarks: Rare; new record from Bandhavgarh National Park.

\section{Pachyrrhinadoretus frontatus (Burm.)}

1855. Adoretus frontatus Burm., Handb. Ent., iv, I, p. 530.

1912. Pachyrrhinadoretus costatus Ohaus, Deutsche Ent. Zeits., p. 511.

Material examined: Umaria, B.N.P., Tala guest house, 22. ix. 2002 (5 exs.), coll. M.L. Koshta \& party.

Remarks: Rare; new record from Madhya Pradesh.

\section{Subfamily VII: Dynastinae}

38. Heteronychus lioderes Redtenbacher

1867. Heteronychus lioderes Redtenbacher, Reise der Novara, Zool., Col., II: 75

1910. Heteronychus lioderes Arrow, Faun. Brit. India,(Lamellicornia: Dynastinae) I: 295.

Material examined: Umaria, B.N.P., Tala Guest House, 22.ix.2002 (1ex.), 24.ix.2002 (1 ex.), coll. M.L. Koshta \& party.

Remarks: Common; new record from Madhya Pradesh.

\section{Alissonotum simile Arrow}

1910. Alissonotum simile Arrow, Faun. Brit. India, (Lamellicornia: Dynastinae) I: 300.

Material examined: Umaria, B.N.P., Tala Guest House, 22.ix.2002 (1 ex.), coll. M.L. Koshta \& party; Tala Guest house, 31.i.2004 (1 ex.), coll. Dr. Y.N. Gupta \& party.

Remarks: Rare; new record from Madhya Pradesh.

\section{Dipelicus cantator Arrow}

1910. Dipelicus cantator Arrow, Faun. Brit. India, (Lamellicornia: Dynastinae) I: 313.

Material examined: Umaria, B.N.P., Tala Guest House, 22.ix.2002

(1ex.), coll. M.L. Koshta \& party.

Remarks: Rare; new record from Madhya Pradesh

41. Xylotrupes gideon (Linnaeus)

1767. Scarabaeus gideon Linnaeus, Syst. Nat., $12^{\text {th }}$ ed. 1(2): 541. 1910. Xylotrupes gideon Arrow, Faun. Brit. India, (Lamellicornia. Dynastinae) I: 262.

Material examined: Umaria, B.N.P., Tala Guest House, 28.ix.2002 (2 exs.), coll. R.K. Singh \& party.

Remarks: Rare; new record from Bandhavgarh National Park.

\section{Subfamily: Cetoniinae}

42. Clinteria klugi (Hope)

1831. Cetonia klugi, Hope, Gray's Zool. Misc., 25.

1910. Clinteria klugi Arrow, Faun. Brit. India, (Lamellicornia: Cetoniinae) I: 187.

Material examined: Umaria, B.N.P., Tala Guest House, 22.ix.2002 (1 ex.); $24 . i x .2002$ (2 exs.), 26.ix.2002 (2 exs.), coll. M.L. Koshta \& party; Gohari, 30.ix.2002 (1 ex.), coll. R.K. Singh \& party.

Remarks: Very common; new record from Bandhavgarh National Park.

\section{Chiloloba acuta (Wied)}

1823. Cetonia acuta Wied., Zool. Mag., II, 1: 8.

1910. Chiloloba acuta Arrow, Faun. Brit. India, (Lamellicornia: Cetoniinae) I: 172.

Material examined: Umaria, B.N.P., Tala Guest House, 24.ix.2002 (1 ex.), coll. M.L. Koshta \& party; Tala Guest house, 30.ix.2002 (2 exs.), coll. R.K. Singh \& party.

Remarks: Common; new record from Bandhavgarh National Park.

\section{Oxycetonia versicolor (Fabricius)}

1775. Cetonia versicolar Fabricius, Syst. Ent.,: 51

1910. Oxycetonia versicolar: Arrow, Faun. Brit. India, (Lamellicornia: Cetoniinae) I: 164.

Material examined: Umaria, B.N.P., Tala Guest House, 24.ix.2002 (1 ex.), coll. M.L. Koshta \& party; 28.ix.2002 (1 ex.), coll. M.L. Koshta \& party.

Remarks: Common; new record from Bandhavgarh National Park.

\section{REFERENCES}

Arrow, G.J. (1910). The Fauna of British India including Ceylon and Burma Col. Lamellicornia, I (Cetoniinae \& Dynastinae). Taylor \& Francis London, VXIV, 322pp.

Arrow, G.J. (1917). The Fauna of British India including Ceylon and Burma. Col. Lamellicornia, II (Rutelinae) Taylor and Francis, London, V-XIII, $387 \mathrm{pp}+7 \mathrm{fig}+5 \mathrm{pls}$

Arrow, G.J. (1931). The Fauna of British India including Ceylon and Burma Col. Lamellicornia, III (Coprinae). Taylor and Francis, London XII, $428 \mathrm{pp}+61$ figs+19pls, map.

Balthasar, V. (1964). Monographie der Scarabaeidae and Aphodiidae der Palaearktischen and Orientalischaen region Prague, Verl. Tschsl. Akad. Wissensch. III, 652pp+224figs.

Chandra, K. (2000). Inventory of Scarabaeid beetles (Coleoptera) from Madhya Pradesh, India. Zoo's Print Journal 15(11): 359-362.

Chandra, K. and R.K. Singh (2004). On a collection of Scarabaeid beetles from Pachmarhi Biosphere Reserve, Madhya Pradesh. Records of Zoological Survey of India 102(3-4): 43-46.

Kuijten, P.J. (1978). Revision of the Genus Hybosorus Macleay (Coleoprtera: Scarabaeidae: Hybosorinae). Zool. Verh. Leiden, 203: 1-49.

Kuijten, P.J. (1983). Revision of the Genus Phaeochrous Castelnau (Coleoprtera: Scarabaeidae: Hybosorinae). Zool. Verh. Leiden, 165: 1-40.

Machatschke, J.W. (1972). Coleopteran Catalogue (W. Junk), Scarabaeoidea, Melolonthinae: Rutelinae, 239pp

\section{ACKNOWLEDGEMENTS}

The authors are grateful to Dr. J.R.B. Alfred, Director, Zoological Survey of India for providing the necessary facilities. Thanks are also due to the Field Director and other forest officials of Bandhavgarh National Park for the necessary cooperation during the survey work. The Authors are also thankful to the officers and scientific staff of the regional station for the collection of specimens.

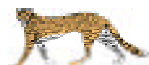

